\title{
Referral Pattern of Patients to Psychiatry Department at Neuroscience Institute in Bangladesh
}

\author{
MM Jalal Uddin'1, MS Jahirul Hoque Chowdhury², Md. Jahidul Islam³, Tauhidul \\ Islam Chowdhury ${ }^{4}$, Morshed Baqui ${ }^{5}$, Poritosh Kumar Sarker, AKM Golam Kabir7, \\ M Badrul Alam ${ }^{8}$, Quazi Deen Mohammad ${ }^{9}$

\begin{abstract}
${ }^{1}$ Assistant Professor, Dept. of Psychiatry, National Institute of Neurosciences and Hospital, Dhaka, Bangladesh; ${ }^{2}$ Assistant Professor, Department of Clinical Neurology cum Resident Physician, National Institute of Neurosciences and Hospital, Dhaka, Bangladesh;

${ }^{3}$ Assistant Professor, Dept. of Physical Medicine and Rehabilitation, National Institute of Neurosciences and Hospital, Dhaka,

Bangladesh; ${ }^{4}$ Assistant Professor, Dept. of Neurology, National Institute of Neurosciences and Hospital, Dhaka, Bangladesh;

${ }^{5}$ Assistant Professor, Dept. of Neurology, National Institute of Neurosciences and Hospital, Dhaka, Bangladesh;

${ }^{6}$ Assistant Professor, Dept. of Neurology, National Institute of Neurosciences and Hospital, Dhaka,

Bangladesh; ${ }^{7}$ Assistant Professor, Dept. of Neuro-Radiology and Imaging, National Institute of Neurosciences and Hospital, Dhaka, Bangladesh; ${ }^{8}$ Joint Directory, Professor of Neurology, National Institute of Neurosciences and Hospital, Dhaka, Bangladesh;

${ }^{9}$ Professor, Director, Professor of Neurology, National Institute of Neurosciences and Hospital, Dhaka, Bangladesh
\end{abstract}

Received on: 24 July 2014; Reviewed on: 26 September 2014; Published on: 1 January 2015

\begin{abstract}
Background: Significant proportion of psychiatric illnesses has been reported in neurology inpatients and outpatients. Objective: The purpose of the present study was to find out the referral pattern of patients to psychiatry department from different departments of National Institute of Neurosciences and Hospital (NINS\&H) and to make diagnosis of referred patients. Methodology: This descriptive cross sectional study was done in psychiatry department at NINS\&H, Dhaka from July 2013 to December 2013. Patients referred to psychiatry department from different outpatients and inpatients departments were taken as study population. Psychiatric diagnoses of the patients were done by the psychiatrist following Diagnostic and Statistical Manual of Mental Disorders-IV-Text Revision (DSM-IV-TR) diagnostic criteria. Semi structured questionnaire was used to collect socio-demographic data. Ethical issues were maintained strictly. Results: A total number of 477 patients were referred to psychiatry department. Most of the patients 469 $(98.32 \%)$ were referred from outpatients department and only $8(1.68 \%)$ from inpatients department. Among the referred patients $97.28 \%$ had psychiatric illness. The commonest psychiatric diagnosis were somatoform disorders $156(32.70 \%)$, anxiety disorders 66(13.83\%), major depressive disorder 95(19.91\%), bipolar mood disorder 27(5.66\%), schizophrenia and related disorders 33(6.92\%). Next common diagnosis were substance related disorder 18(3.77\%), epilepsy 14(2.94\%), mental retardation 13(2.73\%), autism $10(2.10 \%)$, attention deficit hyperactivity disorder $9(1.89 \%)$, sleep disorder $9(1.89 \%)$. Conclusion: In conclusion there is a large number of psychiatric patients attended at the OPD as well as the IPD of the referral neurological tertiary care hospital of which somatoform disorders is the most commonly found. [J Natl Inst Neurosci Bangladesh 2015;1(1): 8-11]
\end{abstract}

Keywords: Referral pattern, psychiatry, neurology

Corresponding author: Dr. MM Jalal Uddin, Assistant Professor, Department of Psychiatry, National Institute of Neurosciences and Hospital, Sher-E-Bangla Nagar, Agargaon, Dhaka-1207, Bangladesh; Email: jalal29march@yahoo.com; Cell No: +8801716772020

Conflict of interest: No conflict of interest with any of the authors.

Funding of the study: None

Contribution of the authors: MMJU, MSJHC, MJI, TIC, MB, PKS, AKMGK, MBA, QDM were contributed from protocol preparation to data collection and have prepared the manuscript and revised by them.

How to cite this article: Uddin MMJ, Chowdhury MSJH, Islam MJ, Chowdhury TI, Baqui M, Sarker PK, Kabir AKMG, Alam MB, Mohammad QD. Referral Pattern of Patients to Psychiatry Department in National Institute of Neurosciences and Hospital, Dhaka. J Natl Inst Neurosci Bangladesh 2015;1(1): 8-11

\section{Introduction}

Neurological illness has been found associated with psychiatric condition and psychological problems are known to occur as a consequence of neurological disorders. High levels of psychiatric illness have been reported in neurology outpatients and inpatient. It has also been shown that psychiatric illnesses are occasionally missed in neurological patients. Bridges and Goldberg reported 39\% of psychiatric illness in neurology inpatients and $72 \%$ of psychiatric illness was unrecognized ${ }^{1,2}$. Carson et al found prevalence of $47 \%$ for anxiety and depressive disorders in patients referred to general neurology outpatient department ${ }^{3}$. Fink et al found that only $1.5 \%$ of the patients with a mental illness were referred to a psychiatrist. Almost 
in every physical illness there is a psychological component in being ill of a person ${ }^{4}$. The significance of assessing the psychiatric aspect of the patients is very important for many reasons. First, the physical illness may directly influence brain and cause psychiatric symptoms. Secondly, the disease may indirectly cause psychological reaction and produce psychiatric symptoms. Thirdly, some psychiatric disorders may present with physical or somatic symptoms. Fourthly, many patients visit to neurology outpatient and may admitted in inpatient department suffer from com-morbid psychiatric illness ${ }^{5}$. Different studies showed that neurological illnesses have been recognized associated with psychiatric conditions and psychological problems are known to occur as a result of neurological disease ${ }^{6-8}$. So it is important therefore further evaluation should be carried out on this aspect. Most of the highly prevalent neurological disorders show a remarkable of co morbidity with specific psychiatric symptoms. Up to $50 \%$ of all patients with stroke develop a depression. Depression also occurs in 55\% of all cases of epilepsy and $40 \%$ of all Parkinson's disease. Also, while $53 \%$ of multiple sclerosis patients suffer from mood disorder ${ }^{9}$. So psychiatric referral is often needed for complete management of the patients and referrals are made when the treating physician has questioned about a patient mental health. The objectives of this study were to find out the referral pattern of patients to psychiatry department from different departments in NINS\&H and to make diagnosis of the referred cases with their sociodemographic characteristics.

\section{Methodology}

This descriptive cross sectional study was in psychiatry department of National Institute of Carrie out, Dhaka from July 2013 to December 2013. All the patients of both sexes referred from the different disciplines both out-patients and inpatients departments were included in the study. Any duplication was excluded from the study. Psychiatric diagnoses were done by the psychiatrist using DSM-IV-TR diagnostic criteria. Semi-structured questionnaire was used to collect the socio-demographic characteristics. Ethical implication was maintained strictly. NINS\&H is a 300 bedded tertiary level neuroscience hospital started from September 2012 situated in Sher-E-Bangla Nagar, Dhaka nearby several hospitals and institutes. There are several departments including clinical neurology, intervention neurology, pediatric neurology, clinical neurosurgery, pediatric neurosurgery, neuro-trauma surgery, gama knife surgery, physical medicine and rehabilitation, psychiatry, cardiology and other allied disciplines such as neurophysiology, pathology, microbiology, biochemistry, pharmacology, neuro radiology and imaging. Intensive care unit (ICU), high dependency unit (HDU) and emergency services also available in this hospital. Patients from all over the country come to NINS\&H and approximately 400 patients come outpatient department every day.

\section{Results}

A total number of 477 patients were referred to the psychiatry department during the study period. Their referral pattern and characteristics are given below using tables and figures.

Table 1: Age distribution of the respondents $(\mathrm{N}=477)$

\begin{tabular}{lcc}
\hline Age (Years) & Frequency & Percentage \\
\hline Less than 20 years & 99 & 20.75 \\
20 to 40 years & 252 & 52.83 \\
40 to 60 years & 95 & 19.92 \\
More than 60 years & 31 & 6.50 \\
Total & $\mathbf{4 7 7}$ & $\mathbf{1 0 0 . 0}$ \\
\hline
\end{tabular}

Table 1 showed the age distribution of the patients and found most of them 252(52.83\%) were 21 to 40 years of age.

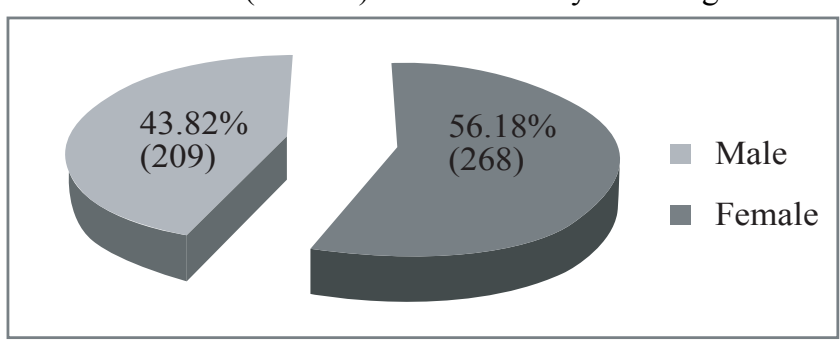

Figure 1: Sex distribution $(\mathrm{N}=477)$

The above figure showed that $56.18 \%$ of the referred patients were male and $43.82 \%$ were female

Table 2: Department wise distribution $(\mathrm{N}=477)$

\begin{tabular}{lccc}
\hline Department & OPD & IPD & Total \\
\hline Neurology & $448(98.7 \%)$ & $6(1.3 \%)$ & $454(100.0 \%)$ \\
P. Neurology & $18(94.7 \%)$ & $1(5.3 \%)$ & $19(100.0 \%)$ \\
Neurosurgery & $3(75.0 \%)$ & $1(25.0 \%)$ & $4(100.0 \%)$ \\
Total & $\mathbf{4 6 9 ( 9 8 . 3 \% )}$ & $\mathbf{8 ( 9 1 . 7 \% )}$ & $\mathbf{4 7 7 ( 1 0 0 . 0 \% )}$ \\
\hline
\end{tabular}

* P. Neurology $=$ paediatric neurology

Most of the referred patients $448(93.92 \%)$ were from neurology OPD. Among the rest 18(3.77\%) were from paediatric neurology OPD, $3(0.63 \%)$ were from neurosurgery department OPD, 6(1.26\%) from neurology IPD, 1(0.21\%) from neurosurgery and IPD of paediatric neurology.

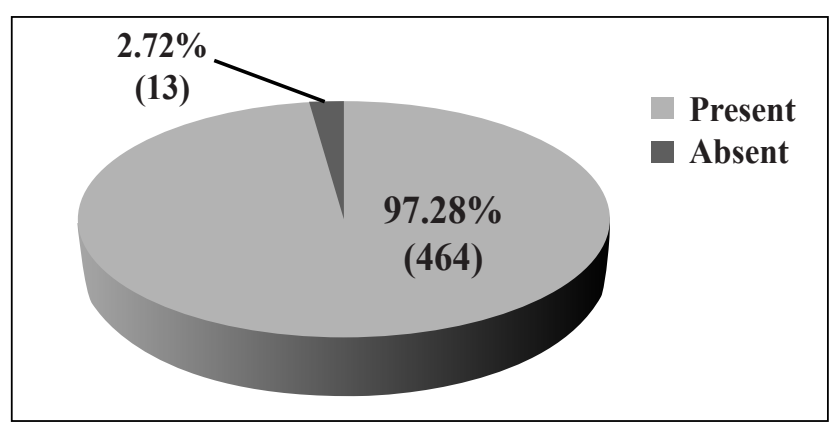

Figure 2: Presence or absence of psychiatric diagnosis $(\mathrm{N}=477)$ 
Among the referred patients 464(97.28\%) had psychiatric illness.

Table 3: Psychiatric diagnosis $(n=464)$

\begin{tabular}{lcc}
\hline Diagnosis & Frequency & Percentage \\
\hline Somatoform disorder (SD) & & \\
$\quad$ Conversion disorder & 28 & 5.87 \\
$\quad$ Pain disorder & 92 & 19.29 \\
$\quad$ Undifferentiated SD & 21 & 4.40 \\
$\quad$ Others & 15 & 3.14 \\
Anxiety disorders & & \\
$\quad$ Phobic disorder & 8 & 1.68 \\
Panic disorder & 12 & 2.52 \\
Obsessive compulsive disorder (OCD) & 27 & 5.66 \\
Generalized anxiety disorder (GAD) & 15 & 3.14 \\
$\quad$ Others & 4 & 0.84 \\
Major depressive disorder (MDD) & 95 & 19.91 \\
Schizophrenia & 24 & 5.03 \\
Delusional disorder & 7 & 1.47 \\
Post partum psychosis & 2 & 0.42 \\
Bipolar mood disorder (BMD) & 27 & 5.66 \\
Substance related disorder (SRD) & 18 & 3.77 \\
Epilepsy & 14 & 2.94 \\
Mental Retardation (MR) & 13 & 2.73 \\
Autistic spectrum disorders (ASD) & 10 & 2.10 \\
Attention deficit hyperkinetic disorder (ADHD) & 9 & 1.89 \\
Sleep disorder & 9 & 1.89 \\
Personality disorder & 5 & 1.05 \\
Total & $\mathbf{4 6 4}$ & $\mathbf{9 7 . 2 8}$ \\
\hline Among the referrd patient & $924(25.99 \%)$ \\
\hline
\end{tabular}

Among the referred patients 124(25.99\%) had co-morbid physical illness.

\section{Discussion}

This descriptive cross sectional study was done in psychiatry department in NINS\&H to see the referral pattern and to make psychiatric diagnosis of the referred patients. Age distribution showed that most 252(52.83\%) of the referred patients were between 20 to 40 years of age group. Similar findings have been reported by Aghanwa et $\mathrm{al}^{10}$ in a study from West Africa found that most of the patients who were referred to psychiatry department from neurology were 16 to 45 years group ${ }^{10}$. In this study above 65 years population was found $6.5 \%$ which was also consistent with another study in Nepal found $8 \%$ of referred patients were above 60 years age group. Among the respondents most of them were male 268(56.18\%) and rest 209(43.18\%) were female. Bhogale et al found male patients were more than female referred patients which was consistent with our study $^{12}$. Reverse findings were found by Shaikh et $\mathrm{al}^{5}$ in a rural medical college hospital in Bangladesh where $62 \%$ of female and rest were male patients who were referred to psychiatry department and this may be due to that female present their problem through somatic complaints ${ }^{5}$.

In the present study it was found that most of the patients $469(98.32 \%)$ were referred from outpatient department (OPD) and only $8(1.68 \%)$ patients were refereed from
Figure 3: Co morbidity $(\mathrm{N}=477)$

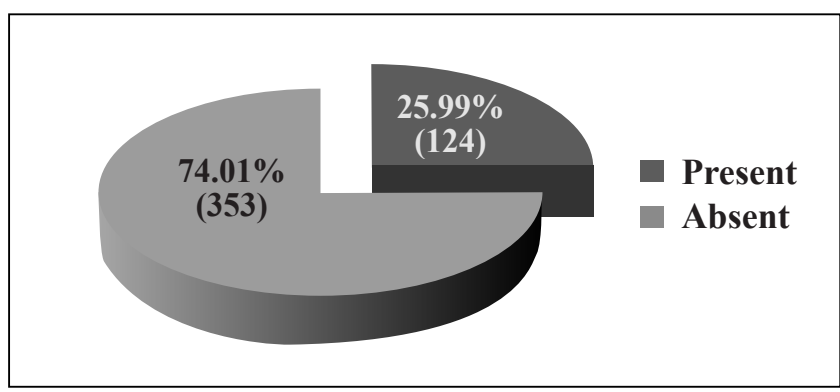

inpatient department (IPD). This may be due to that only selected neurological patients were admitted in IPD and in the OPD patients come for their somatic complaints related to head and in OPD they were seen by the neurologist and when they found no organic cause, then patients were referred to psychiatry department. On the other hand there are $16.01 \%$ of adult population and $18.4 \%$ of children has mental illness in Bangladesh ${ }^{13,14}$. Some patients at first visited to medicine specialist or neurologist for their psychiatric symptom due to social stigma or not aware about their psychiatric symptoms. So this huge number of OPD referral may be the reasons. Among the OPD referral most $448(93.92 \%)$ of the patients were referred from neurology department, $18(3.77 \%)$ patients were referred from paediatric neurology department and only 3(0.63\%) patients were referred from neurosurgery department. In IPD $6(1.26 \%)$ patients were referred from neurology department and paediatric neurology and neuro surgery department each was $1(0.21 \%)$ referred patient. In a study in Europe by Jonge et al found $1.8 \%$ of IPD was referred to consultation liaison psychiatry which is consistent in our study ${ }^{9}$.

Table 4: Co-morbid physical illness $(n=124)$

\begin{tabular}{lcc}
\hline Physical illness & Frequency & Percentage \\
\hline Hypertension & 36 & 7.54 \\
Diabetes mellitus & 24 & 5.03 \\
Stroke (CVD) & 11 & 2.30 \\
IHD & 10 & 2.09 \\
Dementia & 8 & 1.68 \\
Parkinson disease & 12 & 2.52 \\
TB meningitis & 2 & 0.42 \\
CKD & 6 & 1.26 \\
Post encephalitis & 2 & 0.42 \\
Hypercholesterolemia & 13 & 2.73 \\
Total & $\mathbf{1 2 4}$ & $\mathbf{2 5 . 9 9}$ \\
\hline
\end{tabular}

The diagnoses of the referred patients were made by DSMIV-TR diagnostic criteria. Among the referred patients 464(97.28\%) had psychiatric illness and $13(2.72 \%)$ were not diagnosed any psychiatric illness and this was due to that they had some psychiatric symptoms but did not fulfill the diagnostic criteria. The most common psychiatric disorders were found somatoform disorders (156, 32.70\%); among them pain disorder was most frequent $(92,19.29 \%)$, followed by conversion disorder $(28,5.87 \%)$, 
undifferentiated somatoform disorder (21, 4.40\%), somatization disorder and somatoform disorder not otherwise specified $(15,3.14 \%)$. This finding was similar with Fink et $\mathrm{al}^{4}$. and found $33.81 \%$ of somatoform disorders in neurology OPD in their study ${ }^{4}$. This result was also consistent with Ahmed et al ${ }^{15}$. Study conducted by Shaikh et $\mathrm{al}^{5}$ in a rural tertiary care medical college hospital in Bangladesh found $16.30 \%$ of somatoform disorders in their study. Perkin in a study of new referral patients to psychiatry department found $26.5 \%$ of somatoform disorders. The second common diagnosis was major depressive disorder $(95,19.9 \%)$ in the study. Almost similar finding was found by Ahmed et $\mathrm{al}^{15}$ are found $25 \%$ of MDD in neurology OPD. Carson et $\mathrm{al}^{3}$ found $27 \%$ of MDD in their study ${ }^{3}$. Williams et $\mathrm{al}^{17}$ found $33 \%$ and Shaikh et $\mathrm{al}^{5}$ found $35 \%$ of MDD in their studies which was higher in relation to the present study. In this study anxiety disorders were found $13.3 \%$; among them obsessive compulsive disorder (OCD) was found 5.66\%, generalized anxiety disorder $3.14 \%$, panic disorder $2.52 \%$, phobic disorder $1.68 \%$ and other anxiety disorders were $0.84 \%$. Almost similar findings (17.66\%) were found by Ahmed et al in their study. Carson et $\mathrm{al}^{3}$ found $20 \%$ of anxiety disorders in their study in neurology OPD. In the present study $5.6 \%$ of bipolar mood disorder and $6.96 \%$ of schizophrenia and related disorders. Ahmed et al ${ }^{15}$ found $3.66 \%$ of psychosis and $3 \%$ of BMD in their study ${ }^{15}$. Other less common disorders were substance related disorders $18(3.77 \%)$, epilepsy $14(2.94 \%)$, mental retardation $13(2.73 \%)$, autistic spectrum disorders 10(2.10\%), ADHD $9(1.89 \%)$, sleep disorders $9(1.89 \%)$ and personality disorders $5(1.05 \%)$. The high prevalence of somatoform disorders, MDD and anxiety disorders may be due to that people think that they have problem in the brain so they visited neuroscience hospital or neurologist first and ultimately they were diagnosed as a psychiatric symptoms or illness. On the other hand major psychiatric illness like schizophrenia, BMD, delusional disorders and post partum psychosis patients also come to neurology hospital because they think that their problem in the brain so neurology hospital is the place for proper management and occasionally guardian thinks that their patients will be cured if they were seen by the neurologists.

In this study co-morbid physical disorders were found $25.99 \%$ of referred patients and among them $10.90 \%$ were found more than one physical illness. Among the physical illness hypertension (7.54\%), diabetes mellitus (5.03\%), stroke $(2.30 \%)$, ischemic heart disease $(2.09 \%)$, parkinson's disease $(2.52 \%)$, tubercular meningitis $(0.42 \%)$, chronic kidney diseases $(1.26 \%)$, Post encephalitis $(0.42 \%)$ and Hyperlipedmia (2.73\%) were found.

Culturel has a significant impact on the way people perceive their illness and seek treatment. The fact that psychiatric illness is still considered stigmatic may be the reason for people seeking neurologist than psychiatrist. There were some limitations of the study. Short duration of the study, small sample size, lack of informants, some patients were missing in the follow up visit, proper referral systems including referral note were not found in all patients.

\section{Conclusion}

Results of the study indicate that interdepartmental interaction is needed to provide proper comprehensive service to patients. It is particularly important to raise awareness regarding the psychological aspect of any illness of a patient. Further broad based study like assessment of psychiatric morbidity among the patients attending neurology hospital is required to have more evidences in this regard.

\section{References}

1. Bridges KW, Goldberg DP. Psychiatric illness in inpatients with neurogical disorders: patients" views on discussion of emotional problems with neurologists. Br Med Journal 1984; 289:656-656

2. Metcalfe R, Firth D, Pollock S et al. Psychiatric morbidity and illness behavior in female neurological inpatients. J Neurol Neurosurg Psychiatry 1988: 51(11): 294-302

3. Carsson A, Rinbauer B, Mac Kanzie, Warlow C, Sharpe M. Neurological diseases, Emotional disorders and disability: they are related: a study of 300 consecutive new referral to a neurological patient department. J Neurol Neurosurg Psychiatry 2000: 68(2):202-206

4. Fink P, Honsen MS, Sondergaard L, Frydenbe M. Mental illness in new neurological patients. J Neurol Neurosurg Psychiatry 2003; 74: 817-819

5. Shaikh MAK, Ali MZ, Islam MS, Mokhlesuzzaman AKM. Study of referral pattern for psychiatric evaluation in psychiatric OPD in a rural tertiary care hospital. KYAMC Journal 2011; 2(1):128-131

6. Lishman WA. Organic psychiatry. Oxford: Blackwell Scientific Publications, 1978

7. Trimble MR. Neuropsychiatry. New York: John Wiley and Sons, 1981

8. Lipowski ZJ. Psychiatric liaison with neurology and neurosurgery. Am J Psychiatry 1972; 129:136-140

9. Jonge PD, Huse JF, Herzog T, Lobo A, Malt U, Opmeer BC, Kuiper B, Krabbendam A. Referral pattern of neurological patients to psychiatric Consultation-Liason Services in 33 European hospitals. Gen Hosp Psychiatry 2001, 23: 152-157

10. Aghanwa HS, Morakimo $\mathrm{O}$ and Alna OF. Consultation-liaison psychiatry in a general hospital in West Africa. East Afr Med Journal 1996: 73(2): 133-136

11. Jhingan HP. Profile of consultation-liaison psychiatry cases in a new medical college hospital in Nepal. Ind J Psychiatry 1997;39(sup)

12. Bhogale GS, Katte RM, Heble SP, Sinha UK, Patil BA. Psychiatric referral in multispeciality hospital. Ind J Psychiatry 2000; 42(2): 188-194

13. Firoz AHM, Karim ME, Alam MF et al. Prevalence, medical care, awareness and attitude towards mental illness in Bangladesh. Bang $\mathrm{J}$ Psychiatry 2006; 20(1):9-36

14. Rabbani MG, Alam MF, Ahmed HU et al. Prevalence of mental disorders, mental retardation, epilepsy and substance abuse in children. Bang J Psychiatry 2009; 23(1):11-52

15. Ahmed J, Shoib S, Dar MM. Psychiatric morbidity in patients attending neurological outpatient department. Int J Med Sci Pub Health 2013; 2(4):950-952

16. Perkin GD. An analysis of 7836 successive new out patient's referral. J Neurol Neurosurg Psychiatry 1989: 52(4): 447-448.

17. Williams LS, Jones WJ, Shen J et al. Prevalence and impact of depression and pain in neurology out patients. J Neurol Neurosurg Psychiatry 2003: 74(11):1587-1589 